\title{
SISTEMAS DE PREVISÃO DE REQUEIMA EM CULTIVOS DE BATATA EM SANTA MARIA, RS (1)
}

\author{
LEOSANE CRISTINA BOSCO $\left({ }^{2}\right)$; ARNO BERNARDO HELDWEIN $\left({ }^{* *}\right)$; ELENA BLUME $\left({ }^{3}\right)$; \\ GUSTAVO TRENTIN $\left({ }^{4}\right)$; EDENIR LUIS GRIMM $\left({ }^{5}\right)$; DIONÉIA DAIANE PITOL LUCAS $\left(^{(6)}\right.$; \\ LUIS HENRIQUE LOOSE $\left({ }^{6}\right)$; SIDINEI ZWICK RADONS $\left({ }^{2}\right)$
}

\begin{abstract}
RESUMO
Os sistemas de previsão constituem alternativa para o controle eficiente da requeima em batata e consequente redução da quantidade de fungicidas utilizados, dos custos de produção e da poluição ambiental. O objetivo deste trabalho foi avaliar os sistemas de previsão de ocorrência de requeima, Blitecast e Prophy, em cultivos de primavera e outono de batata, cultivar Macaca, suscetível a requeima, na região produtora de Santa Maria (RS). Os experimentos foram desenvolvidos na primavera de 2006 e outono de 2007 com a cultivar Macaca. Os dados meteorológicos foram coletados no centro da área experimental com uma estação automática e com psicrômetros instalados em diferentes níveis. Os tratamentos foram arranjados no delineamento inteiramente casualizado, com quatro repetições, sendo diferenciados por meio dos valores de severidade acumulados, calculados pelos sistemas de previsão Blitecast e Prophy. Os valores de severidade acumulados foram utilizados como indicadores do momento de aplicação de fungicida para o controle da requeima. Verificou-se que a utilização do sistema de previsão de requeima Prophy, com acúmulo de 15, 20 ou 25 valores de severidade e do sistema Blitecast com 24 valores de severidade acumulados, para o genótipo de batata suscetível, Macaca, permite reduzir o número de aplicações de fungicidas sem afetar significativamente a produtividade de tubérculos comerciais.
\end{abstract}

Palavras-chave: Phytophthora infestans, Solanum tuberosum, Blitecast, Prophy.

\section{ABSTRACT \\ FORECAST SYSTEMS OF LATE BLIGHT IN POTATO CROPS IN SANTA MARIA, RIO GRANDE DO SUL STATE, BRAZIL}

\begin{abstract}
The forecast systems are an alternative to the efficient control of late blight potatoes and consequent reduction in the amount of fungicide and thereby reduce production costs and environmental pollution. The objective of this study was to evaluate forecast systems, Blitecast and Prophy, for late blight occurrence in Macaca potato cultivar in Santa Maria, Rio Grande do Sul State, Brazil. The experiments were carried out during Spring 2006 and Autumn 2007 with the Macaca cultivar characterized as susceptible to late blight. Meteorological data were collected at the center of the experimental area with an automatic station and with psicrometers installed at different heights. The treatments were arranged in a completely randomized design, with four replications. Treatments were differentiated by using accumulated values of severity, which were calculated by the Blitecast and Prophy forecasting systems. The values of severity accumulated had the purpose of indicating the time of fungicide application for late blight control. The use of the forecast system of late blight, Prophy, with accumulation of 15, 20 or 25 values of severity, and system Blitecast with 24 accumulated severity for a potato susceptible genotype, Macaca, reduces the number of applications of fungicides without affecting productivity.
\end{abstract}

Key words: Phytophthora infestans, Solanum tuberosum, Blitecast, Prophy

(1) Recebido para publicação em 27 de agosto de 2008 e aceito em 15 de março de 2010.

(2) Estudante do Programa de Pós-graduação em Agronomia, Universidade Federal de Santa Maria (UFSM). Bolsista CAPES.

(3) Centro de Ciências Rurais (CCR), UFSM, 97105-900 Santa Maria (RS), Brasil. E-mail: heldwein@ccr.ufsm.br.

(*) Autor correspondente. Bolsista CNPq.

(4) Estudante do Programa de Pós-graduação em Agronomia, UFSM. Bolsista CNPq.

$\left.{ }^{5}\right)$ Estudante do Programa de Pós-graduação em Engenharia Agrícola, UFSM. Bolsista CNPq.

${ }^{6}$ ) Estudante do curso de graduação em Agronomia, CCR, UFSM. Bolsista PIBIC/CNPq/UFSM. 


\section{INTRODUÇÃO}

A batata é a quarta cultura em ordem de importância agrícola no mundo, depois do trigo, arroz e milho, sendo um dos principais alimentos da humanidade, e representa um dos produtos vegetais frescos de maior preferência na dieta da população brasileira (FAOSTAT, 2008). A batata não é a principal cultura agrícola do Brasil, mas mesmo assim o país é classificado como um dos maiores produtores da América Latina, com colheita recorde de 3,1 milhões de toneladas em 2006. Nos últimos 15 anos, a produção brasileira de batata cresceu $75 \%$ e os rendimentos médios aumentaram de 14 para $22 \mathrm{t} \mathrm{ha}^{-1}$.

Apesar desse crescimento, a cultura da batata é afetada por vários fatores que depreciam a produtividade nas regiões produtoras do Brasil, constituindo-se a ocorrência de requeima (Phytophthora infestans (Mont.) de Bary) o de maior importância. Essa doença é também relatada como o principal problema na maioria dos países que cultivam batata no mundo e, em muitos deles, leva ao uso indiscriminado de fungicidas. Como consequência, tem causado aumento no custo de produção e no impacto ambiental, devido à contaminação da água e do solo (ERWin e RibEIRO, 1996; GARCíA, 2005). Devido à importância da doença como fator limitante à cultura da batata, diferentes métodos de controle integrado têm sido usados, como por exemplo, a utilização de tubérculos-sementes sadios, cultivares menos suscetíveis, retirada e destruição de plantas voluntárias e controle químico (TAYLOR et al., 2003). Atualmente, considera-se que todas as cultivares comerciais são suscetíveis a alguma raça do patógeno, pois $P$. infestans, por meio da reprodução sexuada, é capaz de formar novas raças fisiológicas. Portanto, o método mais eficiente para o controle da requeima ainda é o químico (KIRK et al., 2005).

Nos últimos anos, o controle da requeima aumentou os custos de produção da cultura da batata, alcançando, no Brasil, 10\% do seu valor total (AgrianUAL, 2007). As aplicações de fungicidas são, em geral, excessivas por serem realizadas de forma empírica, sem considerar a interação entre fatores biológicos do ciclo de vida do patógeno e as condições meteorológicas. Assim, alternativas para controle químico mais racional precisam ser consideradas, tais como a utilização dos sistemas de previsão de ocorrência da doença. Esses sistemas consideram diferentes elementos meteorológicos, sendo os mais usados a temperatura média do ar, o número de horas com umidade relativa do ar acima de 90\%, o tempo de molhamento foliar e a precipitação pluvial.

Nesses sistemas, os períodos de condições favoráveis à doença são determinados por meio declasses de condições meteorológicas utilizadas como critérios, índices ou modelos que descrevem o desenvolvimento da doença, os quais servem de indicativo do momento mais adequado para as aplicações de fungicidas. Dos diversos sistemas de previsão para a requeima em uso, alguns determinam somente o momento da primeira aplicação de fungicida. Outros sistemas, como o Blitecast (Krause et al., 1975) e o Prophy (SCHEPERS, 1995), determinam as aplicações necessárias para o controle da requeima durante todo o ciclo de cultivo, através de um conjunto de regras que relacionam os elementos meteorológicos condicionantes para sua evolução.

Os sistemas de previsão são considerados ferramentasimportantes para o manejo de doenças e para racionalizar o uso de fungicidas (REIs, 2004; HeldweIN, 2007). A maioria dos sistemas de previsão utilizados para a requeima da batata foram desenvolvidos em regiões temperadas e devem ser ajustados quando forem utilizados em regiões subtropicais e tropicais. Nas regiões temperadas, os invernos rigorosos afetam a sobrevivência de $P$. infestans, bem como a dinâmica de inóculo, devido à ausência de plantas hospedeiras (GARRETT e DENDY, 2001). As condições edafoclimáticas na Região Sul do Brasil são diferentes da maioria das grandes regiões produtoras do mundo, sendo importante conhecer e caracterizá-las especificamente para que se possam utilizar sistemas de previsão de doenças de forma adequada. Os sistemas Blitecast e o Prophy estão sendo avaliados na Região Central do Rio Grande do Sul, onde resultados preliminares (TRENTIN, 2006; GRIMM, 2007) sugerem que poderão ser úteis no controle da requeima, em épocas de cultivo favoráveis à doença, e reduzir o número de aplicações de fungicidas sem prejuízo à produtividade.

Embora os sistemas Blitecast e Prophy já tenham sido avaliados sob condições de campo no Brasil (NAZARENO et al., 1999; ReIs et al., 1999; Costa et al., 2002; Batista et al., 2006; Trentin, 2006; GrimM, 2007), mais experimentos são necessários, considerando diferentes áreas, épocas de cultivo e cultivares para atender as exigências requeridas para a validação dos sistemas de previsão de doença em uma determinada região. Esse fato motivou a realização deste estudo, cujo objetivo foi avaliar os sistemas de previsão de ocorrência de requeima, Blitecast e Prophy, em cultivos de primavera e outono, com a cultivar de batata Macaca, suscetível à requeima, na região produtora de Santa Maria (RS).

\section{MATERIAL E MÉTODOS}

Osexperimentosforam realizados em Santa Maria, RS (latitude de $29^{\circ} 43^{\prime} \mathrm{S}$; longitude de $53^{\circ} 43^{\prime} \mathrm{W}$ e altitude de $95 \mathrm{~m}$ ), localizada na região fisiográfica da Depressão Central do Estado do Rio Grande do Sul. O clima local, segundo a classificação climática de Köppen, pertence ao tipo Cfa, subtropical úmido, com verões quentes e 
sem estação seca definida. As temperaturas médias do ar variam entre $12,9^{\circ} \mathrm{C}$, em junho, e $24,6^{\circ} \mathrm{C}$, no mês de janeiro (HeLDWEIn et al., 2009). O solo do local pertence à unidade de mapeamento São Pedro, classificado como Argissolo Vermelho distrófico arênico (EMBRAPA, 2006).

A cultivar Macaca foi utilizada na primavera de 2006 (23/ 8 a 14 / 12) e no outono de 2007 (15/3 a 21 / 6) por ser amplamente cultivada nessas duas épocas na Região Central do Rio Grande do Sul e devido à precocidade de brotação, ciclo curto e suas características para fins culinários. No entanto, ela é suscetível a requeima sob condições favoráveis a essa doença e exige aplicações de fungicidas durante todo seu ciclo. O manejo das plantas seguiu as recomendações técnicas para o cultivo da batata no Rio Grande do Sul (Bisognin, 1996).

Para controle da requeima, com exceção das parcelas testemunha, foram utilizados os fungicidas mancozebe (Dithane NT - 3,0 $\mathrm{kg} \mathrm{ha}^{-1}$ do produto comercial), oxicloreto de cobre (Cuprogarb $350-4 \mathrm{~g}$ $\mathrm{L}^{-1}$ de água do produto comercial), piraclostrobina + metiram (Cabrio Top - 3,0 $\mathrm{kg} \mathrm{ha}^{-1}$ do produto comercial), conforme recomendações do Ministério da Agricultura, Pecuária e Abastecimento (MAPA, 2007). A aplicação dos produtos foi realizada de forma alternada entre os produtos de ação de contato (Dithane NT e Cuprogarb) e o de ação sistêmica (Cabrio Top), observando-se os valores de severidade acumulados para cada tratamento. Para a aplicação, foi utilizado um pulverizador costal de 20 litros de capacidade, ao qual foi acoplado um bico de pulverização do tipo cone vazio.

Dois sistemas de previsão de requeima foram avaliados: Blitecast (KraUSE et al., 1975) e Prophy (SCHEPERS, 1995). No sistema Blitecast, os tratamentos foram programados conforme o acúmulo de valores de severidade (VS), que são o resultado da combinação entre a umidade relativa e temperatura média do ar no período em que a umidade relativa do ar for maior que 90\% (UR $>90 \%$ ), ambas medidas a 1,5 m do nível do solo, ou seja, acima do dossel das plantas de batata. Os valores de severidade foram calculados diariamente com base nas classes de temperatura média do ar durante os períodos contínuos de UR $>90 \%$, conforme tabela 1.
O sistema Prophy estabelece que a primeira aplicação de fungicida para cultivares suscetíveis é realizada no momento que o cultivo alcança a altura de $0,15 \mathrm{~m}$. As aplicações subsequentes foram programadas conforme o acúmulo de valores de severidade, calculados a partir de dados de pares psicrômetros não-ventilados, constituídos de sensores de resistência elétrica de platina (Pt-100). Os dados foram coletados com frequência de 1s e médias foram armazenadas a cada 10 minutos em um aquisitor eletrônico de dados (datalogger), entre as $12 \mathrm{~h}$ de um dia e às $11 \mathrm{~h} 59 \mathrm{~min}$ do dia seguinte. Nesse sistema, o cálculo do acúmulo de valores de severidade também foi realizado com base nos dados de umidade relativa e temperatura do ar (Tabela 1). No entanto, esses dados foram medidos por sensores instalados a $0,1 \mathrm{~m}$ do nível do solo, conforme critério do sistema.

Para monitorar os gradientes de temperatura e umidade relativa do ar no perfil vertical do dossel, foram montadas torres com pares psicrométricos nos dois níveis exigidos pelos sistemas de previsão. Em cada época de cultivo, foram utilizadas duas repetições de pares psicrométricos. Os dados obtidos nas duas repetições de dois níveis de medidas psicrométricas foram agrupados através de médias e a partir das médias de temperatura do bulbo seco e de bulbo úmido foram obtidos os valores de pressão de saturação de vapor do ar, pressão parcial de vapor do ar e umidade relativa do ar.

No sistema Blitecast, os valores de severidade diários foram acumulados até o momento em que atingiam 18 e 24 VS (tratamentos BLI18 e BLI24 respectivamente) e para o sistema Prophy, os valores de severidade diários foram acumulados até 15,20 e $25 \mathrm{VS}$ (tratamentos PRO15, PRO20 e PRO25, respectivamente) (Tabela 2). Ao atingirem-se esses VS, aplicava-se o fungicida nas plantas do respectivo tratamento, iniciando-se em zero a nova contagem de valores de severidade.

Para verificar a acurácia dos sistemas de previsão em prever a ocorrência da requeima por meio de variáveis meteorológicas, foram realizadas avaliações de severidade da doença utilizando-se a escala diagramática de JAMES (1971). As avaliações de severidade foram

Tabela 1. Classes de temperatura média do ar durante os períodos contínuos de acúmulo de horas com umidade relativa maior do que $90 \%$ e respectivos valores de severidade (VS) diários para o controle da requeima nos cultivos de batata. Santa Maria, 2008

\begin{tabular}{lccccc}
\hline Classes de Temperatura & \multicolumn{5}{c}{ Tempo com umidade relativa $>90 \%$} \\
\hline${ }^{\circ} \mathrm{C}$ & $\leq 15$ & $16-18$ & $19-21$ & $22-24$ & \\
$7,2-11,6$ & $\leq 12$ & $13-15$ & $16-18$ & $19-21$ & $>22$ \\
$11,7-15,0$ & $\leq 9$ & $10-12$ & $13-15$ & $16-18$ & $>19$ \\
$15,1-26,6$ & 0 & 1 & 2 & 3 \\
\hline $\mathrm{VS}$ & & & & 4 \\
\hline
\end{tabular}

Fonte: KRAUSE et al. (1975). 
Tabela 2. Tratamentos com diferentes esquemas de aplicação de fungicidas, em função dos valores de severidade (VS) acumulados, calculados pelos Sistemas Blitecast (BLI) e Prophy (PRO) para o cultivo de batata Macaca. Santa Maria, 2008

\begin{tabular}{lccc}
\hline Tratamento & Sistema de Previsão & VS & Descrição \\
\hline TEST & Sem aplicação $\left({ }^{1}\right)$ & - & Testemunha para o clone Macaca \\
Semanal & Semanal $\left({ }^{1}\right)$ & - & Semanal para o clone Macaca \\
PRO15 & Prophy & 15 & Prophy com 15 VS para o clone Macaca \\
PRO20 & Prophy & 20 & Prophy com 20 VS para o clone Macaca \\
PRO25 & Prophy & 25 & Prophy com 25 VS para o clone Macaca \\
BL18 & Blitecast & 18 & Blitecast com 18 VS para o clone Macaca \\
BLI24 & Blitecast & 24 & Blitecast com 24 VS para o clone Macaca \\
\hline
\end{tabular}

(1) Tratamentos em que não foram utilizados os sistemas de previsão.

realizadas nos dois períodos experimentais, em três plantas por parcela. Foram avaliadas trêsfolhas, dispostas na parte inferior, mediana e superior, em cada uma das três plantas na área útil de cada parcela, as quais foram marcadas com um barbante branco assim que eram identificados os sintomas da requeima. A média das três folhas representou a severidade observada na planta, a média das três plantas a severidade da parcela e a média de severidade das quatro parcelas de cada tratamento consistiu a severidade observada para o tratamento.

$\mathrm{Na}$ primavera de 2006, foram realizadas avaliações de severidade aos 51, 55, 59, 63, 67 e 71 dias após a emergência (DAE), enquanto no outono de 2007, as avaliações de severidade ocorreram aos 17, 21, 25, 30, 34, 38, 43, 47, 50, 55, 59, 62, 66 e 70 DAE. Após a obtenção das médias da severidade observada em cada planta marcada, foi calculada a duração da área foliar sadia (DAFS) e a área abaixo da curva de progresso da doença (AACPD), conforme descrito por CAMPBELl e MADDEN (1990). A taxa aparente de infecção da requeima nas folhas em relação ao tempo foi determinada pelo método de regressão linear, após a transformação dos dados de severidade em proporção para logito, de acordo com van DER PLANK (1963). Em virtude das condições do inverno na região não serem extremas o suficiente para eliminar o inóculo do patógeno, não foi realizada inoculação artificial de $P$. infestans, e a infecção do hospedeiro pelo patógeno ocorreu naturalmente nas épocas de cultivo, quando as condições ambientais foram favoráveis.

O acompanhamento dos estágios fenológicos foi realizado durante os dois períodos de cultivo. O ciclo de desenvolvimento da cultura da batata foi dividido em três fases: da emergência ao início da tuberização (EMIT), do início da tuberização ao início da senescência (IT-IS) e do início da senescência à colheita (IS-CO). Na primavera de 2006, o IT ocorreu aos 17 DAE, o IS aos 63 DAE e a CO aos 88 DAE. No outono de 2007 o IT, IS e CO ocorreram aos 17, 50 e 79 DAE respectivamente.

No fim do ciclo da cultura, quando as plantas estavam com as folhas totalmente senescidas, foi realizada a dessecação da parte aérea e dez dias depois, iniciou-se a colheita individual de cada parcela experimental. Os tubérculos colhidos foram classificados em comerciais e não comerciais. Foram considerados tubérculos comerciais aqueles com diâmetro superior a $2,3 \mathrm{~cm}$ (COSTA et al., 2007) e sem incidência de distúrbios fisiológicos e não comerciais os demais tubérculos. A partir desses dados, determinouse o número de tubérculos total (NTT), comerciais (NTC), não comerciais (NTNC) e a massa fresca média de todos os tubérculos (MMT), de tubérculos comerciais (MMTC) e não comerciais (MMTNC). Com os dados de número de tubérculos e massa fresca determinaram-se a produtividade total $(\mathrm{PT})$, produtividade comercial $(\mathrm{PC})$ e produtividade não comercial (PNC) por parcela e por tratamento em $\mathrm{tha}^{-1}$.

Foram utilizados sete tratamentos (Tabela 2) arranjados no delineamento inteiramente casualizado, com quatro repetições. Cada repetição constituiu uma parcela composta por quatro fileiras de plantas, com 5,0 m de comprimento e 3,2 m de largura. Com o objetivo de minimizar as interferências entre parcelas, consideraram-se como parcela útil 3,0 $\mathrm{m}$ de comprimento das duas fileiras centrais, nas quais foram realizadas as avaliações durante os experimentos. A análise dos dados foi realizada considerando-se cada safra de cultivo separadamente com sete esquemas da aplicação de fungicida. Aos resultados aplicou-se análise de variância pelo teste $\mathrm{F}$ e as médias foram comparadas pelo teste de Tukey $(\mathrm{p}<0,05)$. Os dados de severidade final observada (SVFO) foram transformados em $\operatorname{arcsen} \sqrt{\mathrm{x} / 100}$ para análise estatística.

\section{RESULTADOS E DISCUSSÃO}

$\mathrm{Na}$ primavera, a manifestação da requeima ocorreu em duas etapas. Até 50 DAE não se constatou a presença de lesões nas plantas da área experimental em todos os tratamentos (Figura 1) devido, provavelmente, à ausência de inóculo na área. Entre 50 e 55 DAE a evolução da severidade da requeima foi lenta, a partir de então, ocorreu estabilização e/ou paralisação da 

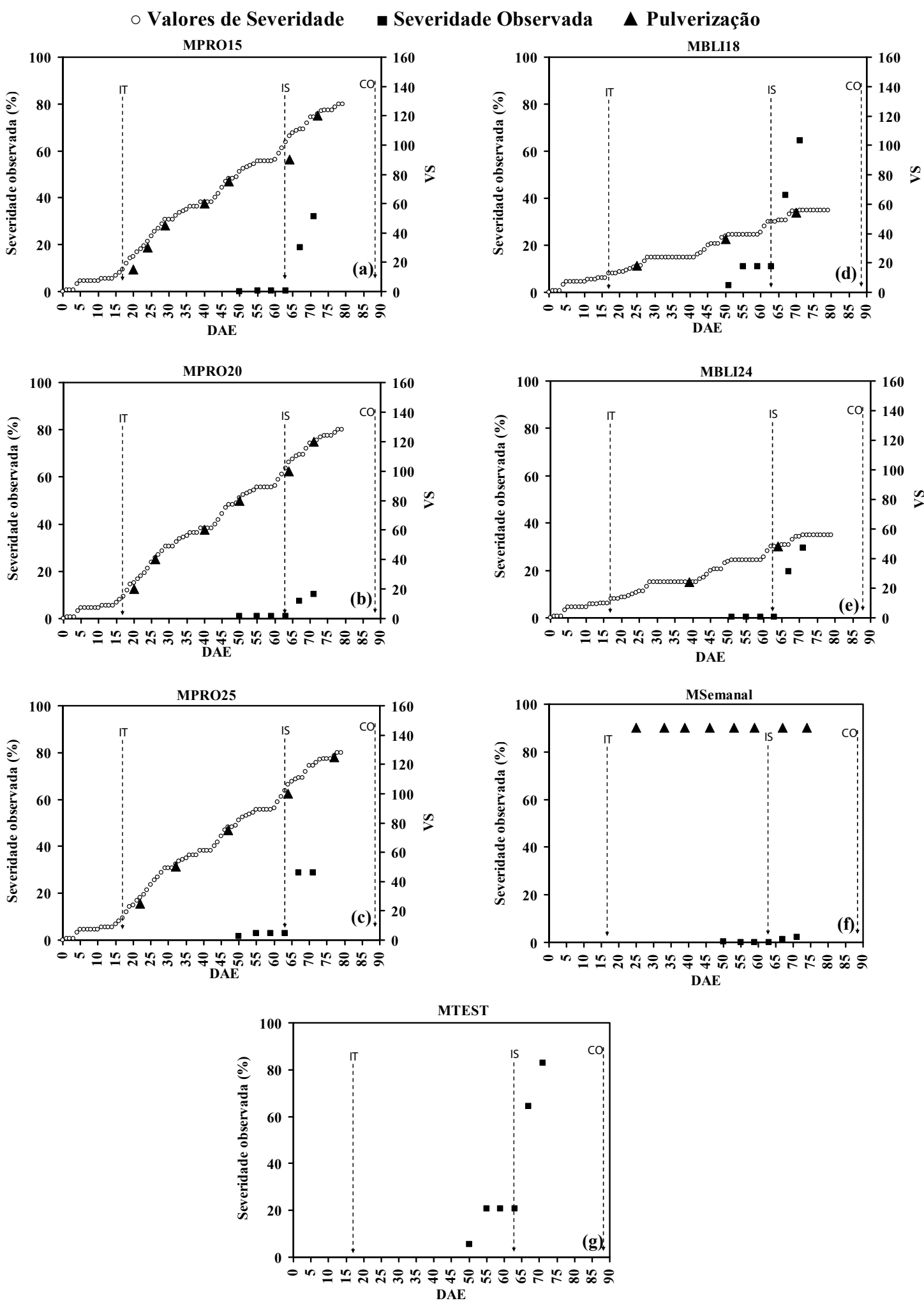

Figura 1. Momentos da pulverização de fungicidas ( $\boldsymbol{\Delta})$, valores de severidade calculados (VS) (o) pelos sistemas de previsão de requeima Prophy $(a, b, c)$ e Blitecast (d, e) e evolução da severidade observada (em \%) de requeima (a) nos tratamentos com o sistema de previsão Prophy com 15 (PRO15), 20 (PRO20) e 25 (PRO25) valores de severidade; com o sistema de previsão Blitecast com 18 (BLI18) e 24 (BLI24) valores de severidade; com pulverizações semanais (Semanal; f) e testemunha (TEST; g) em cultivo de batata, cultivar Macaca, na primavera de 2006. As linhas pontilhadas indicam o início dos estágios de desenvolvimento da cultura: início de tuberização (IT), início de senescência (IS) e colheita (CO). 
taxa de progresso da requeima até 62 DAE. Entre 55 e 62 DAE o período com umidade relativa do ar acima de $90 \%$ foi menor do que $9 \mathrm{~h}$ em seis e quatro dias seguidos, conforme os sistemas Blitecast e Prophy, respectivamente, com temperatura média variando entre 15 e $19{ }^{\circ} \mathrm{C}$ (Figura 3a e 3c). A segunda etapa da manifestação da requeima ocorreu a partir de $66 \mathrm{DAE}$, quando se observou uma evolução rápida na severidade da requeima, principalmente no tratamento testemunha (Figura 1g), indicando que o patógeno resistiu à condição de baixa umidade do ar do período anterior. Em virtude do período determinado como relativamente desfavorável à requeima pelo sistema de previsão (55 a $62 \mathrm{DAE}$, Figura 3a), o intervalo entre as aplicações de fungicida nos tratamentos PRO15 e PRO25 foi de 17 dias e do PRO20 de 13 dias. Este é um intervalo longo que pode ter facilitado a evolução da doença a partir dos 66 DAE caso estivessem infectados anteriormente. Devido às condições desfavoráveis, não houve manifestação das frutificações, mas poderia haver uma evolução lenta do micélio nos tecidos, principalmente sob a presença de orvalho (VAN EIMERN, 1964).

No tratamento PRO20, o intervalo entre as aplicações de fungicidas foi menor, sendo um pouco mais eficiente no controle da requeima do que nos tratamentos PRO15 e PRO25 (Tabela 3). Nos tratamentos BLI18 e BLI24, foram realizadas menos pulverizações durante o ciclo da cultura do que nos demais tratamentos, no entanto, não necessariamente ocasionou severidade de requeima maior nas plantas dos tratamentos BLI24, onde ocorreu severidade equivalente ao PRO15 e PRO25, com diferença estatística apenas da testemunha (Tabela 3). No tratamento semanal (Figura 1f), praticamente, não se observou severidade de requeima, pois os intervalos entre as pulverizações foram de apenas sete dias e proporcionaram proteção quase absoluta à cultura durante seu ciclo de desenvolvimento.

$\mathrm{Na}$ época de cultivo de outono, os primeiros sintomas da requeima foram identificados aos $21 \mathrm{DAE}$
(Figura 2). Observou-se que esses sintomas apareceram distribuídos nas plantas, com alguns na parte basal, outros na parte mediana e também superior das plantas. As folhas basais foram destruídas mais rapidamente pela requeima, em virtude do prolongado período com umidade relativa acima de 90\% (Figura 3) se comparadas às folhas superiores. Observou-se a presença de solo nas folhas, resultante de salpicos ocasionados pela chuva e o contato de folhas com o solo devido ao acamamento das plantas. Segundo Huber e GILlespie (1992) e Heldwein et al. (2007), em noites com orvalho, ou em dias de chuva há presença de água livre sobre as folhas e, dessa forma, os esporângios podem ser produzidos. Se o molhamento ocorrer por tempo prolongado, os esporos germinam sobre a planta ainda durante o mesmo período de molhamento, podendo infestar novamente o tecido vegetal. No outono ocorreram 66 dias com condições de umidade relativa acima de $90 \%$ por períodos superiores a 10 horas diárias. Outro fator importante, favorável a requeima, foi que a temperatura do ar se manteve acima de $16{ }^{\circ} \mathrm{C}$ na maior parte do ciclo (Figura 3d). Segundo ERWIN e RIBEIRO (1996), a temperatura ótima para a requeima está entre 16 e $23{ }^{\circ} \mathrm{C}$. Essas constatações confirmam que as condições para o patógeno infectar a planta e esporular existiram durante todo o ciclo da cultura durante o outono e foram previstas ou determinadas tanto pelo sistema Prophy quanto pelo Blitecast.

Observou-se que a evolução da doença, no outono, ocorreu lentamente até fim da tuberização (50 DAE). A partir desse momento, que corresponde ao estágio de início de senescência, a evolução foi rápida até o fim do ciclo da cultura (colheita). A taxa aparente de infecção da requeima na fase de início de tuberização (17 $\mathrm{DAE})$ até início da senescência (50 DAE) variou de 0,1 (Semanal, PRO15, PRO20, PRO25, BLI24) a 0,2 (TEST e BLI18), não havendo diferença significativa entre as taxas dos tratamentos. Na fase de início da senescência até a colheita (79 DAE) a taxa aparente de infecção foi de 0,1 (Semanal e PRO20), 0,2 (TEST, PRO15, PRO25 e BLI24) e

Tabela 3. Dias de área foliar sadia (DAFS), área abaixo da curva de progresso da doença (AACPD) e severidade final observada (SVFO) para os fatores época de cultivo e esquema de aplicação de fungicida para a cultivar de batata Macaca. Santa Maria, 2008

\begin{tabular}{|c|c|c|c|c|c|c|}
\hline \multirow{2}{*}{$\begin{array}{l}\text { Esquema } \\
\text { de aplicação }\end{array}$} & \multicolumn{2}{|c|}{ DAFS } & \multicolumn{2}{|c|}{ AACPD } & \multicolumn{2}{|c|}{ SVFO } \\
\hline & Primavera & Outono & Primavera & Outono & Primavera & Outono \\
\hline TEST & $16,1 \mathrm{~B}$ & $33,5 \mathrm{~B}$ & $682,0 \mathrm{~A}$ & $1618,7 \mathrm{~A}$ & $82,8 \mathrm{~A}$ & $100,0 \mathrm{~A}$ \\
\hline Semanal & $22,9 \mathrm{~A}$ & $40,7 \mathrm{AB}$ & $10,5 \mathrm{~B}$ & $713,6 \mathrm{BC}$ & $2,2 \mathrm{C}$ & $55,9 \mathrm{CD}$ \\
\hline PRO15 & $21,8 \mathrm{~A}$ & $45,4 \mathrm{~A}$ & $142,6 \mathrm{~B}$ & $162,5 \mathrm{C}$ & $32,0 \mathrm{BC}$ & $30,5 \mathrm{D}$ \\
\hline PRO20 & $22,4 \mathrm{~A}$ & $42,0 \mathrm{~A}$ & $62,9 \mathrm{~B}$ & $584,0 \mathrm{BC}$ & $10,3 \mathrm{C}$ & $56,0 \mathrm{D}$ \\
\hline PRO25 & $21,2 \mathrm{~A}$ & $41,5 \mathrm{~A}$ & $185,9 \mathrm{AB}$ & $655,4 \mathrm{BC}$ & $28,6 \mathrm{BC}$ & $69,4 \mathrm{BCD}$ \\
\hline BLI18 & $18,8 \mathrm{AB}$ & $39,9 \mathrm{AB}$ & $430,7 \mathrm{AB}$ & $908,5 \mathrm{ABC}$ & $64,4 \mathrm{AB}$ & $95,7 \mathrm{AB}$ \\
\hline BLI24 & $21,8 \mathrm{~A}$ & $38,0 \mathrm{AB}$ & $140,0 \mathrm{~B}$ & $1099,3 \mathrm{AB}$ & $29,4 \mathrm{BC}$ & $93,1 \mathrm{ABC}$ \\
\hline
\end{tabular}

Médias seguidas por mesma letra, na vertical, não diferem entre si pelo teste Tukey a 5\% de probabilidade. PRO15, PRO20, PRO25 = sistema de previsão Prophy com 15, 20 e 25 valores de severidade, respectivamente; BLI18, BLI24 = sistema de previsão Blitecast com 18 e 24 valores de severidade, respectivamente; Semanal = pulverizações semanais; TEST = testemunha. 


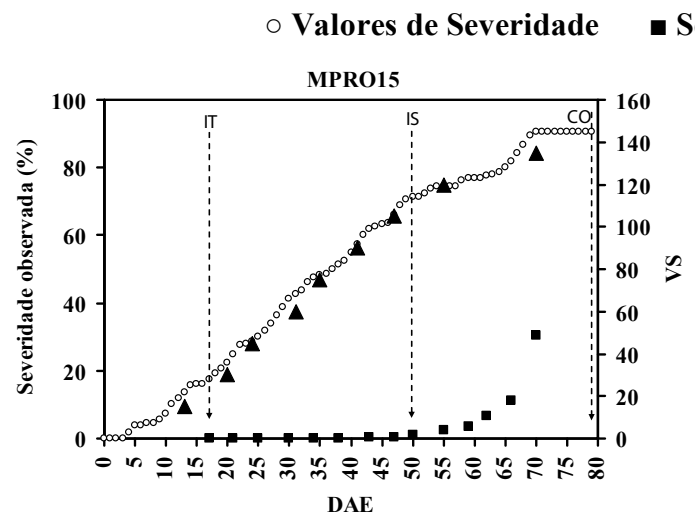

\section{Severidade Observada $\Delta$ Pulverização}
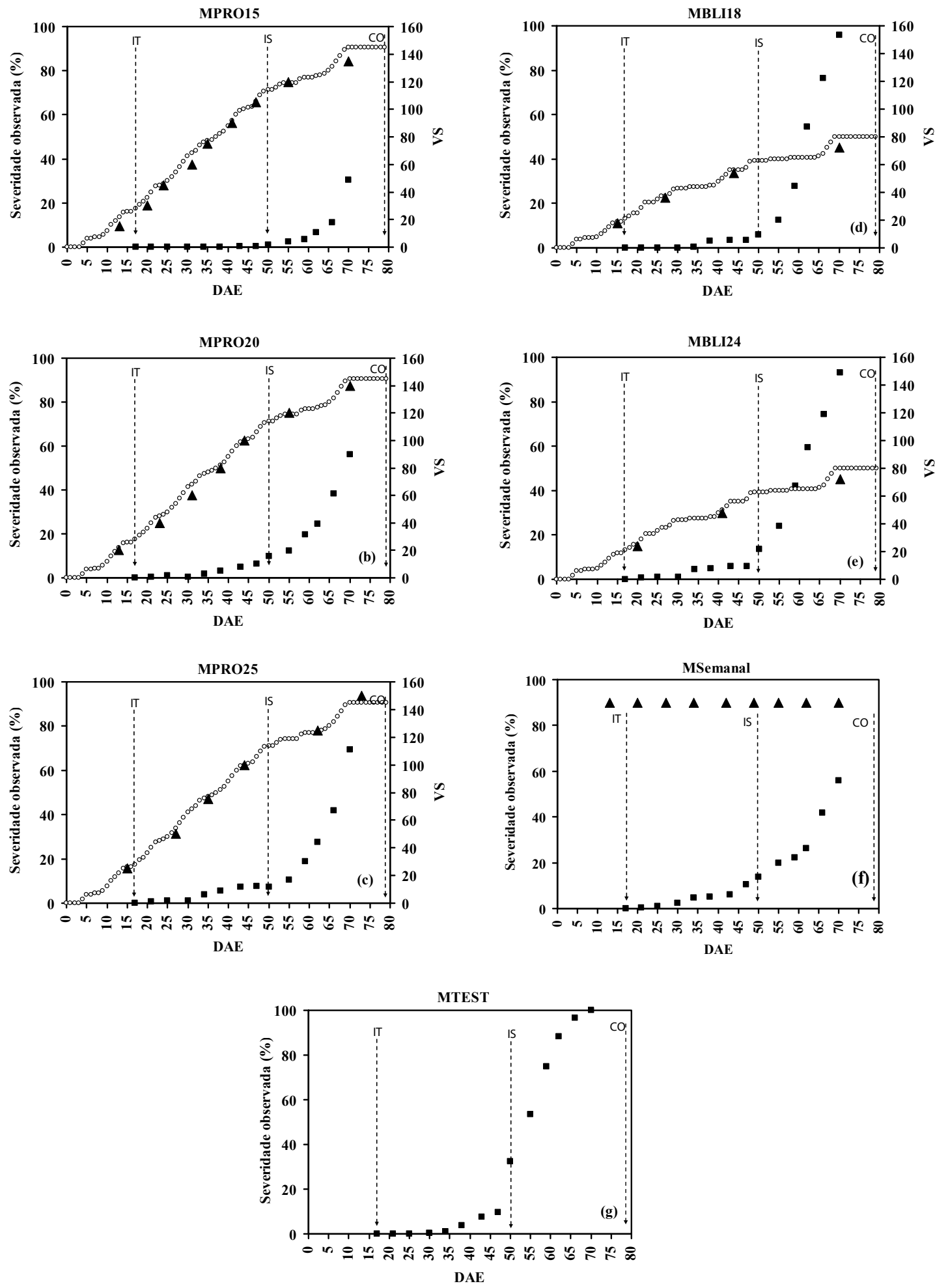

Figura 2. Momentos da pulverização de fungicidas ( $\mathbf{\Lambda}$ ), valores de severidade calculados (VS) (०) pelos sistemas de previsão de requeima Prophy $(\mathrm{a}, \mathrm{b}, \mathrm{c})$ e Blitecast (d, e) e evolução da severidade observada (em \%) de requeima ( $\mathbf{(})$ nos tratamentos com o sistema de previsão Prophy com 15 (PRO15), 20 (PRO20) e 25 (PRO25) valores de severidade; com o sistema de previsão Blitecast com 18 (BLI18) e 24 (BLI24) valores de severidade; com pulverizações semanais (Semanal; f) e testemunha (TEST; g) em cultivo de batata, cultivar Macaca, no outono de 2007. As linhas pontilhadas indicam o início dos estágios de desenvolvimento da cultura: início de tuberização (IT), início de senescência (IS) e colheita (CO). 

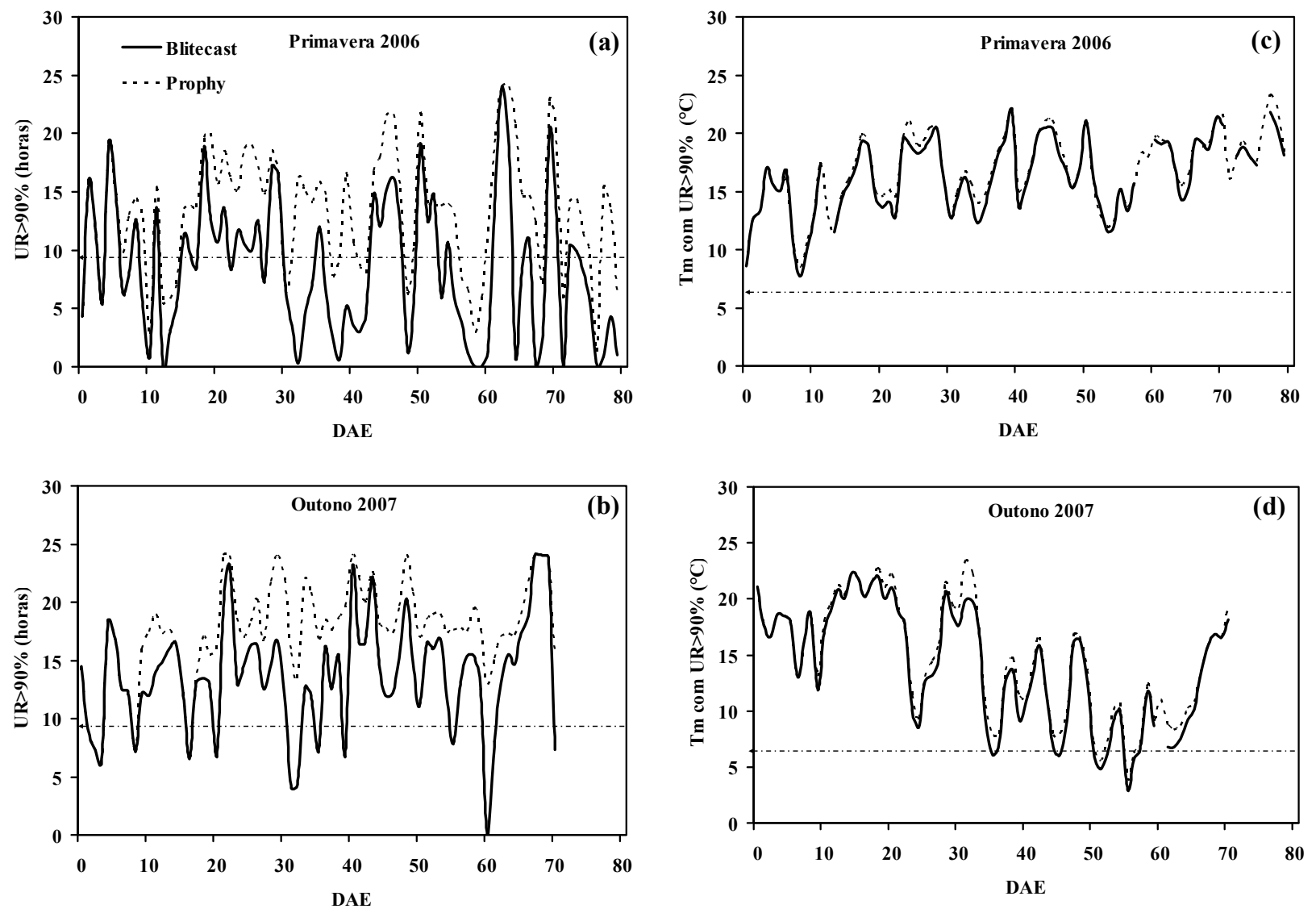

Figura 3. Período de tempo em horas com umidade relativa do ar maior do que $90 \%$ (UR>90\%) e temperatura média do ar (Tm) nos períodos com umidade relativa do ar maior do que $90 \%$ verificados ao utilizar os sistemas Blitecast e Prophy durante a primavera de 2006 e o outono de 2007 para a cultivar Macaca, em função do número de dias após a emergência (DAE). A linha pontilhada indica o mínimo de temperatura $\left(7,2^{\circ} \mathrm{C}\right)$ e de duração do período com UR $>90 \%$ (9 horas) para que ocorra o desenvolvimento da requeima.

de 0,3 (BLI18). Percebe-se, portanto, que nos tratamentos PRO15, PRO25, BLI18 e BLI24 a taxa de infecção foi maior na fase de início da senescência até a colheita.

A partir de 50 DAE, observou-se um intervalo maior entre as aplicações de fungicida, quando comparado aos intervalos das aplicações anteriores, tanto nos tratamentos com o sistema de previsão Prophy quanto no sistema Blitecast. Esse fato significa que no período entre 50 e $65 \mathrm{DAE}$ as condições do ambiente (temperatura ou umidade relativa do ar acima de $90 \%$ ) não foram propícias ao acúmulo de valores de severidade pelos sistemas, proporcionando maiores intervalos entre as aplicações. No entanto, o oomiceto Phytophthora infestans (Mont.) de Bary estava presente nas plantas e continuou ativo, mesmo sem as condições ótimas para seu desenvolvimento.

Onúmero de pulverizações do tratamento semanal determinadas por intervalo fixo de sete dias foi o mesmo do tratamento PRO15 e com duas e três pulverizações a mais do que o PRO20 e PRO25 respectivamente(Figura 2).
No entanto, no esquema Semanal a severidade provocada pela requeima foi estatisticamente igual à severidade observada nos tratamentos PRO15, PRO20, PRO25 e BLI24 (Tabela 3). Mesmo havendo redução do número de pulverizações, as plantas foram protegidas com os esquemas de aplicação do sistema Prophy e Blitecast, pois com o monitoramento adequado das condições do ambiente, o momento em que as pulverizações foram realizadas foi mais adequado e representativo em relação à ocorrência da doença conforme afirmam CosTA et al. (2002); TAYLOR et al. (2003) e HeLDWEIN et al. (2007). Essas constatações são importantes, pois asseguram que as aplicações de fungicidas devem ser realizadas com base nas condições do ambiente e não com intervalos fixos entre elas, além de mostrar que há possibilidade de redução da poluição ambiental a partir da redução de aplicações de fungicidas.

Entre os sistemas de previsão utilizados, constatouse que o sistema Blitecast previu um menor acúmulo de VS do que o Prophy durante todo o ciclo da cultura nos 
dois períodos experimentais (Figura 2a,b), concordando com os resultados de Trentin (2006). O sistema de previsão Prophy, em geral, previu a primeira aplicação de fungicida mais cedo do que o sistema Blitecast, semelhante ao observado em trabalhos desenvolvidos na Europa (HANSEN et al., 2001), nos quais foram analisados seis diferentes sistemas de previsão para a requeima e o Prophy recomendou a primeira aplicação de fungicida antes que os demais, além de um número maior de aplicações. Essas diferenças ocorreram, principalmente, devido à posição de instalação dos psicrômetros. Os sensores para o sistema Prophy estavam a 0,1 m do solo, medindo a umidade relativa do ar no interior do dossel de plantas, local em que a umidade permanece elevada por um período mais prolongado do que acima do dossel de plantas (VAN EIMERN, 1964; HeLDWEin, 1993). Por esse motivo, o sistema de previsão Prophy acumulou mais valores de severidade em um menor período, preconizando mais aplicações de fungicidas do que o sistema Blitecast.

Pelos valores de umidade relativa do ar dos diferentes níveis de medida confirma-se essa condição, pois se constatou que, tanto em dias com chuva quanto em dias sem chuva, ela é superior no interior do dossel das plantas (Figura 3). Em experimentos realizados com as culturas de milho e de soja, Pedro Júnior e GILlesPie $(1982 a, b)$ verificaram que não havia diferenças entre as medidas de umidade relativa do ar realizadas dentro ou fora do dossel das plantas. Assumiram que os valores de pressão parcial de vapor no dossel eram iguais aos medidos em uma estação automática fora do dossel para a estimativa de duração do molhamento. No entanto, HeLDWEIN (1993) verificou que para a cultura da batata existem diferenças de umidade relativa e pressão parcial de vapor medida dentro e acima do dossel das plantas e que essas são condicionadas pela diferença da pressão parcial de vapor e podem ser maiores do que 3,0 hPa; a maior diferença ocorre das $18 \mathrm{~h}$ às $22 \mathrm{~h}$, período em que, geralmente, inicia-se a formação do orvalho. Além disso, dentro do abrigo meteorológico e acima de um dossel de batata, observa-se que o comportamento normal da pressão parcial de vapor possui dois pontos de máxima, sendo um no entardecer e o outro, na maioria das vezes, no fim da manhã (BERGER-LANDEFELDT et al., 1956; VAN EIMERN, 1964), enquanto no interior do dossel o pico de máxima do entardecer quase nunca ocorre. O pico de máxima da pressão parcial de vapor acima do dossel no entardecer ocorre principalmente porque a velocidade do vento decresce rapidamente com a estabilização do ar junto à superfície, enquanto no interior do dossel os processos de transpiração das plantas e, principalmente, de evaporação na superfície do solo ainda contribuem para o aumento da pressão parcial de vapor (HELDWEIN, 1993).

$\mathrm{Na}$ análise estatística para as variáveis que caracterizam a quantidade de doença (DAFS, AACPD e SVFO) ocorrida em cada tratamento verificaram-se diferenças principalmente com relação ao tratamento TEST, tanto na primavera de 2006 quanto no outono de 2007 (Tabela 3). O DAFS e AACPD foram semelhantes entre os tratamentos nas duas épocas de cultivo, e os tratamentos com o sistema Prophy e Semanal tiveram, em termos relativos, mais dias de área foliar sadia do que nos tratamentos com o sistema Blitecast, em virtude do menor ataque da requeima expresso em AACPD e SVFO. Entre os tratamentos com aplicação de fungicida na primavera de 2006, verificou-se que no tratamento BLI18 ocorreu maior severidade, porém diferiu apenas do Semanal e PRO20. No outono de 2007, observaramse menores severidades nos tratamentos PRO15, PRO20, PRO25 e Semanal (Tabela 3).

$\mathrm{Na}$ análise estatística para as variáveis referentes aos componentes de produtividade, verificou-se que na primavera de 2006 os tratamentos com aplicação de fungicida praticamente não interferiram na produtividade comercial (PC), não comercial (PNC) e total (PT), bem como no número e na massa de tubérculos (Tabelas 4 e 5). O tratamento testemunha teve produtividade semelhante aos tratamentos com

Tabela 4. Produtividade comercial (PC), produtividade não comercial (PNC) e produtividade total (PT), em função do esquema de aplicação de fungicida em cada safra para a cultivar de batata Macaca. Santa Maria, 2008

\begin{tabular}{lcccccc}
\hline \multirow{2}{*}{$\begin{array}{l}\text { Esquema de } \\
\text { aplicação }\end{array}$} & \multicolumn{2}{c}{ PC } & \multicolumn{2}{c}{ PNC } & \multicolumn{2}{c}{ PT } \\
\cline { 2 - 6 } & Primavera & Outono & Primavera & Outono & Primavera \\
\cline { 2 - 7 } TEST & $7,2 \mathrm{AB}$ & $6,4 \mathrm{~B}$ & $3,7 \mathrm{~A}$ & $0,9 \mathrm{~A}$ & $10,9 \mathrm{AB}$ & $7,3 \mathrm{~B}$ \\
Semanal & $11,2 \mathrm{~A}$ & $11,9 \mathrm{AB}$ & $5,1 \mathrm{~A}$ & $2,9 \mathrm{~A}$ & $16,3 \mathrm{~A}$ & $14,8 \mathrm{AB}$ \\
PRO15 & $6,2 \mathrm{~B}$ & $14,5 \mathrm{~A}$ & $3,7 \mathrm{~A}$ & $2,1 \mathrm{~A}$ & $9,9 \mathrm{~B}$ & $16,6 \mathrm{~A}$ \\
PRO20 & $5,6 \mathrm{~B}$ & $13,4 \mathrm{AB}$ & $4,2 \mathrm{~A}$ & $1,6 \mathrm{~A}$ & $1,8 \mathrm{~B}$ & $10,2 \mathrm{~B}$ \\
PRO25 & $6,0 \mathrm{~B}$ & $11,4 \mathrm{AB}$ & $4,2 \mathrm{~A}$ & $1,4 \mathrm{~A}$ & $12,8 \mathrm{AB}$ \\
BLI18 & $5,7 \mathrm{~B}$ & $10,3 \mathrm{AB}$ & $3,5 \mathrm{~A}$ & $1,7 \mathrm{~A}$ & $9,2 \mathrm{~B}$ & $12,0 \mathrm{AB}$ \\
BLI24 & $9,3 \mathrm{AB}$ & $10,6 \mathrm{AB}$ & $4,7 \mathrm{~A}$ & $2,2 \mathrm{~A}$ & $14,0 \mathrm{AB}$ & $12,8 \mathrm{AB}$ \\
\hline
\end{tabular}

Médias seguidas por letras iguais, maiúsculas na vertical não diferem entre si pelo teste Tukey a 5\% de probabilidade. PRO15, PRO20, PRO25 = sistema de previsão Prophy com 15, 20 e 25 valores de severidade, respectivamente; BLI18, BLI24 = sistema de previsão Blitecast com 18 e 24 valores de severidade, respectivamente; Semanal = pulverizações semanais; TEST = testemunha. 
Tabela 5. Número total de tubérculos (NTT), número de tubérculos não comerciais (NTNC), massa média de tubérculo (MMT) e massa média de tubérculo comercial (MMTC) em cada safra para a cultivar de batata Macaca. Santa Maria, 2008

\begin{tabular}{|c|c|c|c|c|c|c|c|c|}
\hline \multirow{3}{*}{$\begin{array}{l}\text { Esquema de } \\
\text { aplicação }\end{array}$} & \multicolumn{2}{|c|}{ NTT } & \multicolumn{2}{|c|}{ NTNC } & \multicolumn{2}{|c|}{ MMT } & \multicolumn{2}{|c|}{ MMTC } \\
\hline & Primavera & Outono & Primavera & Outono & Primavera & Outono & Primavera & Outono \\
\hline & \multicolumn{8}{|c|}{$\mathrm{N} .^{\circ} \mathrm{g}$} \\
\hline TEST & $128 \mathrm{~A}$ & $47 \mathrm{~A}$ & $77 \mathrm{~A}$ & $15 \mathrm{~A}$ & $35,5 \mathrm{~A}$ & $35,6 \mathrm{C}$ & $58,5 \mathrm{~A}$ & $46,4 \mathrm{C}$ \\
\hline Semanal & $168 \mathrm{~A}$ & $74 \mathrm{~A}$ & $100 \mathrm{~A}$ & $26 \mathrm{~A}$ & $38,3 \mathrm{~A}$ & $61,5 \mathrm{AB}$ & $65,4 \mathrm{~A}$ & $67,3 \mathrm{ABC}$ \\
\hline PRO15 & $131 \mathrm{~A}$ & $59 \mathrm{~A}$ & $81 \mathrm{~A}$ & $17 \mathrm{~A}$ & $36,5 \mathrm{~A}$ & $65,8 \mathrm{~A}$ & $60,8 \mathrm{~A}$ & 79,3A \\
\hline PRO20 & $115 \mathrm{~A}$ & $76 \mathrm{~A}$ & $70 \mathrm{~A}$ & $22 \mathrm{~A}$ & $40,8 \mathrm{~A}$ & $57,8 \mathrm{ABC}$ & $60,5 \mathrm{~A}$ & $71,1 \mathrm{AB}$ \\
\hline PRO25 & $121 \mathrm{~A}$ & $74 \mathrm{~A}$ & $77 \mathrm{~A}$ & $18 \mathrm{~A}$ & $40,6 \mathrm{~A}$ & $51,3 \mathrm{ABC}$ & $66,8 \mathrm{~A}$ & $59,7 \mathrm{ABC}$ \\
\hline BLI18 & $117 \mathrm{~A}$ & $68 \mathrm{~A}$ & $71 \mathrm{~A}$ & $23 \mathrm{~A}$ & $38,0 \mathrm{~A}$ & $47,4 \mathrm{ABC}$ & $59,9 \mathrm{~A}$ & $61,3 \mathrm{ABC}$ \\
\hline BLI24 & $154 \mathrm{~A}$ & $98 \mathrm{~A}$ & $90 \mathrm{~A}$ & $40 \mathrm{~A}$ & $43,5 \mathrm{~A}$ & $41,2 \mathrm{BC}$ & $69,3 \mathrm{~A}$ & $54,6 \mathrm{BC}$ \\
\hline
\end{tabular}

Médias seguidas por mesma letra, na vertical, para cada fator, não diferem entre si pelo teste Tukey a 5\% de probabilidade. PRO15, PRO20, PRO25 = sistema de previsão Prophy com 15, 20 e 25 valores de severidade, respectivamente; BLI18, BLI24 = sistema de previsão Blitecast com 18 e 24 valores de severidade, respectivamente; Semanal = pulverizações semanais; TEST = testemunha.

fungicidas com maior produtividade (Semanal e BLI24). Na primavera, o surgimento dos sintomas da requeima ocorreu no fim do estágio de tuberização, não interferindo no acúmulo de assimilados pelos tubérculos.

No outono, observou-se que a PC, a PT e a MMT foram diferentes entre os tratamentos, porém o número de tubérculos foi semelhante (Tabelas 4 e 5). A requeima ocorrida no período de outono de 2007 interferiu no acúmulo de massa dos tubérculos, pois ocorreu durante a fase de tuberização (Figura 2). TRENTIN (2006) afirma que a partir do início da tuberização não há alteração no número de tubérculos, mas sim no seu tamanho. No tratamento PRO15, a produtividade comercial foi de $5,5 \mathrm{tha}^{-1}$ superior à testemunha e estatisticamente não diferiu dos demais tratamentos. No entanto foi de 2,6 e 3,9 $\mathrm{t} \mathrm{ha}^{-1}$ superior aos tratamentos Semanal e BLI24, respectivamente, evidenciando que apesar de não haver diferença significativa as plantas foram mais bem protegidas pelo tratamento PRO15. O tratamento BLI24 recebeu o menor número de aplicações de fungicidas no outono (três pulverizações), com apenas 29,4\% de severidade de requeima e não teve a produtividade (PC e PT) comprometida significativamente em relação ao tratamento semanal e PRO15 (nove pulverizações) (Tabela 4). Esse fato significa que a cultura da batata necessita de aplicações de fungicidas para evitar perdas devido à requeima; no entanto, o uso dos sistemas de previsão indicam com maior eficiência os momentos mais adequados para aplicação dos fungicidas, uma vez que consideram a interação patógeno e ambiente. Outra constatação na comparação entre os tratamentos com aplicação de fungicida é a de que o tratamento BLI18 apresentou menor produtividade, não diferindo da TEST no outono de 2007. Esse tratamento, que havia preconizado quatro aplicações de fungicidas, uma a mais do que o BLI24, não foi eficiente com relação ao controle da requeima.
É importante salientar que a área foliar sadia é indispensável para a fotossíntese e acúmulo de fotoassimilados, sendo por esse motivo fator determinante da produção das culturas. No entanto, em ambiente de campo outros elementos além das aplicações de fungicidas que protegem a área foliar podem influenciar na produtividade da cultura. Segundo Pereira (1987), teores mais altos de matéria seca podem ser atribuídos também ao fotoperíodo e à temperatura que, segundo BEUKEMA e VAN DER ZAAG (1990), influenciam no crescimento das plantas e na formação dos tubérculos.

\section{CONCLUSÃO}

A utilização do sistema de previsão de requeima Prophy, com acúmulo de 15, 20 ou 25 valores de severidade e do sistema Blitecast com 24 valores de severidade acumulados, para o genótipo de batata suscetível, Macaca, permite reduzir o número de aplicações de fungicidas sem afetar significativamente a produtividade de tubérculos comerciais.

\section{REFERÊNCIAS}

AGRIANUAL 2007. Anuário da Agricultura Brasileira. São Paulo: Instituto FNP, 2007. 516p.

BATISTA, D.C.; LIMA, M.A.; HADDAD, F.; MAFFIA, L.A.; MIZUBUTI, E.S.G. Validation of decision support systems for tomato early blight and potato late blight, under Brazilian conditions. Crop Protection, v.25, p.664-670, 2006.

BERGER-LANDEFELDT, U.; KIENDL, J.; DANNENBERG, H. Beobachtungen über das Temperatur und Dampfdruckunruhe über Pflanzenbeständen. Meteorogischer Rundschau, v.90, p.120-130, 1956.

BEUKEMA, H.P.; VAN DER ZAAG, D.E. Introduction to potato production. 2.ed. Wageningen: PUDOC, 1990. 224p. 
BISOGNIN, D.A. Recomendações técnicas para o cultivo da batata no Rio Grande do Sul e Santa Catarina. Santa Maria: UFSM, 1996. 63p.

CAMPBELL, C.L.; MADDEN, L.V. Forecasting plant diseases. In: CAMPBELL, C.L.; MADDEN, L.V. (Ed.). Introduction to plant disease epidemiology. New York: John Wiley, 1990. p.423-452.

COSTA, L.C.; BISOGNIN, D.A.; ANDRIOLO, J.L.; RITTER, C.E.L.; BANDINELLI, M.G. Identificação de clones de batata com potencial para mesa e adaptados para os cultivos de outono e primavera do Rio Grande do Sul. Ciência e Natura, v.29, p.93-104, 2007.

COSTA, R.V.; ZAMBOLIM, L.; VALE, F.X.R.; MIZUBUTI, E.S.G. Previsão da requeima da batateira. Fitopatologia Brasileira, v.27, p.349-354, 2002.

EMBRAPA (Empresa Brasileira de Pesquisa Agropecuária). Centro Nacional de Pesquisa de Solos. Sistema brasileiro de classificação de solos. 2.ed. Rio de Janeiro: Embrapa Solos, 2006. 306p.

ERWIN, D.C.; RIBEIRO, O.K. Phytophthora: Diseases Worldwide. St. Paul, Minnesotta: APSPress, 1996. 592p.

FAOSTAT. Potato world. Disponível em: <http: / / faostat.fao. org >. Acesso em: 17 jan. 2008.

GARCÍA, B.I.L. Risco climático de ocorrência da requeima da batata na região dos Andes, Venezuela. 2005. 132 f. Tese (Doutorado em Agronomia) - Escola Superior Agricultura “Luiz de Queiroz"-USP, Piracicaba.

GARRETT, K.A.; DENDY, S.P. Cultural practices in potato late blight management. In: GILB LATIN AMERICAN WORKSHOP, 1, 2001. Proceedings... Cochabamba: GILB publications, 2001, p.107-114.

GRIMM, E.L. Efeito de diferentes níveis de irrigação na produtividade e ocorrência de Requeima na cultura da batata. 2007. 70 f. Dissertação (Mestrado em Engenharia Agrícola) Universidade Federal de Santa Maria - UFSM, Santa Maria.

HANSEN, J.G. et al. Results of validation trials of Phytophthora DSSs in Europe, 2001. In: WORKSHOP OF A EUROPEAN NETWORK FOR DEVELOPMENT OF AN INTEGRATED CONTROL STRATEGY OF POTATO LATE BLIGHT, 6, 2001, Wageningen. Proceedings... Wageningen: PPO-Special Report, 2001. p.231-242.

HELDWEIN, A.B. Ermittlung der Taubenetzung von Pflanzenbeständen durch Anvendung mikrometeorologischer Verfahren sowie mittels konventioneller Methoden. 1993. $206 \mathrm{f}$. Tese(Doktor der Agrarwissenschaften)-FachbereichInternationale Agrarentwicklung, Tu-Berlin.

HELDWEIN, A.B.; WILSMANN, S.; TAZZO, I.F.; NIED, A.H. Índicesbiometeorológicosemonitoramentoagrometeorológico de doenças e pragas. In: CARLESSO, R. (Ed.). Uso e benefícios da coleta automática de dados meteorológicos na agricultura.
Santa Maria: Universidade Federal de Santa Maria, 2007, p.135-155.

HELDWEIN, A.B.; BURIOL, G.A.; STRECK, N.A. O clima de Santa Maria. Ciência \& Ambiente, v.1, p.43-58, 2009.

HUBER, L.; GILLESPIE, T.J. Modeling leaf wetness in relation to plant disease epidemiology. Annual Review of Phytopathology, v.30, p.553-577, 1992.

JAMES, W.C. An illustrated series of assessment keys for plant diseases, their preparation and usage. Cannadian Plant Disease Survey, v.51, p.39-65, 1971.

KIRK, W.W.; ABU-EL SAMEN, F.M.; MUHINYUZA, J.B.; HAMMERSCHMIDT, R.; DOUCHES, D.S.; THILL, C.A.; GROZA, H.; THOMPSON, A.L. Evaluation of potato late blight management utilizing host plant resistance and reduced rates and frequencies of fungicide applications. Crop Protection, v.24, p.961-970, 2005.

KRAUSE, R.A.; MASSIE, L.B.; HYRE, R.A. BLITECAST: a computerized forecast of potato late blight. Plant Disease Reporter, v.59, p.95-98, 1975.

MAPA (Ministério da Agricultura, Pecuária e Abastecimento). Coordenação-Geral de Agrotóxicos e Afins/DFIA/DAS. Disponível em: < http: / / www.agricultura.gov.br/ >. Acesso em: 10 nov. 2007.

NAZARENO, N.R.X.; SCOTTI, C.A.; MAFIOLETTI, R.L.; BOSCHETTO, N. Controle da requeima da batata através do monitoramento das variáveis climáticas. Fitopatologia Brasileira, v.24, p.170-174, 1999.

PEDRO JUNIOR, M.J.; GILLESPIE, T.J. Estimating dew duration I. Utilizing micrometeorological data. Agricultural and Forest Meteorology, v.25, p.283-296, 1982a.

PEDROJUNIOR, M.J.; GILLESPIE, T.J.Estimating dew duration II. Utilizing standard weather station data. Agricultural and Forest Meteorology, v.25, p.297-310, 1982b.

PEREIRA, A.S. Composição química, valor nutricional e industrialização. In: REIFSCHNEIDER, F. J. B. (Ed.). Produção de batata. Brasília: Linha Gráfica e Editora, 1987. p.12-28.

REIS, E.M.; MEDEIROS, C.A.; CASA, R.T.; MENDEZ, C. Previsão de doenças de plantas: sistemas para a requeima e para a pinta preta da batateira. Summa Phytopathologica, v.25, p.60-65, 1999.

REIS, E.M. Previsão de doenças de plantas. 1.ed. Passo Fundo: UPF, 2004, 316p.

SCHEPERS, H.T.A.M. ProPhy: a computerized expert system for control of late blight in potatoes in the Netherlands. In: INTERNATIONAL PLANT PROTECTION CONGRESS, 13., 1995, Netherlands. Proceedings... Netherlands, 1995. p.948.

TAYLOR, M.C.; HARDWICK, N.V.; BRADSHAW, N.J.; HALL, A.M. Relative performance of five forecasting schemes for 
potato late blight (Phytophthora infestans) I. Accuracy of infection warnings and reduction of unnecessary, theoretical, fungicide applications. Crop Protection, v.22, p.275-283, 2003.

TRENTIN, G. Avaliação de sistemas de previsão de ocorrência de Phytophthora infestans em batata. 2006. 100 f. Dissertação (Mestrado em Agronomia) - Curso de Pós-Graduação em Agronomia, Universidade Federal de Santa Maria - UFSM, Santa Maria.
VAN DER PLANK, J.E. Plant Disease: Epidemics and Control. New York: Academic Press, 1963. 349p.

VAN EIMERN, J. Untersuchungen über das klima in pflanzenbestäden als grunlage einer agrarmeteoroligischen beratung insbesonere für den pflanzenschutz. Berichte des Deutschen Wetterdienstes, n.96, 1964. 13p. 